\title{
Microwave imaging of spinning object using orbital angular momentum
}

\author{
Kang Liu ${ }^{1,2, a)}$, Xiang $\mathrm{Li}^{1}$, Yue $\mathrm{Gao}^{2}$, Hongqiang $\mathrm{Wang}^{1}$, and Yongqiang Cheng ${ }^{1}$ \\ ${ }^{1}$ College of Electronic Science and Engineering, National University of Defense \\ Technology, Changsha 410073, China \\ ${ }^{2}$ School of Electronic Engineering and Computer Science, Queen Mary University of \\ London, London E1 4NS, UK
}

\begin{abstract}
The linear Doppler shift used for the detection of spinning object becomes significantly weaken when the line of sight (LOS) is perpendicular to the object, which will result in the failure of detection. In this paper, a new detection and imaging technique of spinning object is developed. The rotational Doppler phenomenon is observed by using the microwave carrying orbital angular momentum (OAM). To converge the radiation energy on the area where objects might exist, the generation method of OAM beams is proposed based on the frequency diversity principle, and the imaging model is derived accordingly. The detection method of the rotational Doppler shift and the imaging approach of the azimuthal profiles are proposed, which is verified by proof-of-concept experiments. Simulation and experimental results demonstrate that OAM beams can still be used to obtain the azimuthal profiles of spinning objects even when the LOS is perpendicular to the object. This work remedies the insufficiency in existing microwave sensing technology and offers a new solution to the object identification problem.
\end{abstract}

\section{INTRODUCTION}

In conventional sensor systems, the detection of moving objects are mainly based on the well-known linear Doppler phenomenon caused by the translational motion. ${ }^{1,2}$ However, when the line of sight (LOS) is perpendicular to a spinning object, the linear Doppler phenomenon does not exist. ${ }^{3,4}$ Thus, the rotational Doppler phenomenon has been introduced for object identification. ${ }^{5,6}$ Specially, the electromagnetic (EM) wave carrying orbital angular momentum $(\mathrm{OAM})^{7,8}$ can be used to observe the rotational Doppler phenomenon effectively. Hitherto, the relationship between the rotational Doppler shift and the OAM has been formulized and utilized to reveal the geometry and motion of spinning objects. ${ }^{6,9,10}$ Moreover, the rotational Doppler shift can be multiplied by the OAM index, which increases the sensitivity to the rotational velocity. Hence, the OAM beams are used to detect and image the spinning objects in this paper, especially in the situations when the LOS is perpendicular to the object.

In optics, the OAM multiplexing technique has been widely used to increase the system capacity in communications. ${ }^{11-15}$ In radio frequencies, the EM wave with OAM was applied in wireless communications ${ }^{16-18}$ and object imaging, ${ }^{19}$ which provides the prospect for improving the performance of conventional systems. In Refs. 19 and 20, the vortex EM waves were utilized to acquire the high-resolution azimuth information of static objects. Imaging algorithms, including FFT, back projection, ${ }^{20}$ power spectrum estimation (PSD) ${ }^{21}$ and compressive sensing ${ }^{22}$ were proposed to reconstruct the object. High-efficiency OAM-generation approaches were developed by using metasurface, ${ }^{23-25}$ novel OAM antenna ${ }^{26}$

\footnotetext{
a) Author to whom correspondence should be addressed. Electronic mail: liukang1117@126.com.
} 
and antenna array. ${ }^{27}$ To achieve high signal-to-noise ratio in practical imaging scenario, the OAM beams of different modes should be converged in the same direction. In Ref. 28, the concentric-ring array was designed and the corresponding imaging methods were proposed. However, the concentric-ring array system is bulky, complicated and costly for practical implementation. Therefore, the compact OAM-generation method with required beam characteristics for imaging needs to be investigated. Meanwhile, to the best of the authors' knowledge, no published papers deal with spinning object imaging using OAM beams.

In this paper, the frequency diversity method is proposed to generate OAM beams, which can solve the beam divergence problem with only one single-ring array. With this OAM-generation method and the object's rotation feature, the imaging model and signal processing method are different from those in the published papers. ${ }^{20,21}$ Thus, the imaging model of the spinning object is derived and an effective imaging algorithm is proposed. Proof-of-concept experiments are carried out and the imaging results are analyzed as well.

\section{THEORY}

According to the principle of static object imaging using OAM, ${ }^{20}$ the circular array is usually designed to generate the beams due to its flexibility. As aforementioned, to reduce the system complexity, the frequency diversity technique is proposed to generate OAM beams with different modes based on only one single-ring array. The sketch map of the detection and imaging of spinning object is shown in Figure 1. For a uniform circular array (UCA), the antennas are phased with phase difference $\delta \phi=2 \pi l / N$ between two elements, ${ }^{29,30}$ where $l$ denotes OAM mode number (also named topological charge) and $N$ is the number of antennas. For a detection point $P(r, \theta, \phi)$ in the far field, the emitted signal $E_{s}(k, l)$ can be written as ${ }^{20}$

$$
\begin{aligned}
E_{s}(k, l) & =\sum_{n=1}^{N} \frac{e^{-i k\left|r-r_{n}\right|} e^{i l \phi_{n}}}{\left|\boldsymbol{r}-\boldsymbol{r}_{n}\right|} \approx \frac{e^{-i k r}}{r} \sum_{n=1}^{N} e^{i\left(k \cdot r_{n}+l \phi_{n}\right)} \\
& \approx \frac{e^{-i k r}}{r} N i^{l} e^{i l \phi} J_{l}(k a \sin \theta)
\end{aligned}
$$

where $a$ denotes the array radius, $k$ is the wavenumber and $\boldsymbol{r}_{n}\left(a, \phi_{n}\right)$ denotes the position of the $n^{\text {th }}$ antenna on the xoy plane. $J_{l}(k a \sin \theta)$ is the $l^{\text {th }}$ order Bessel function of first kind.

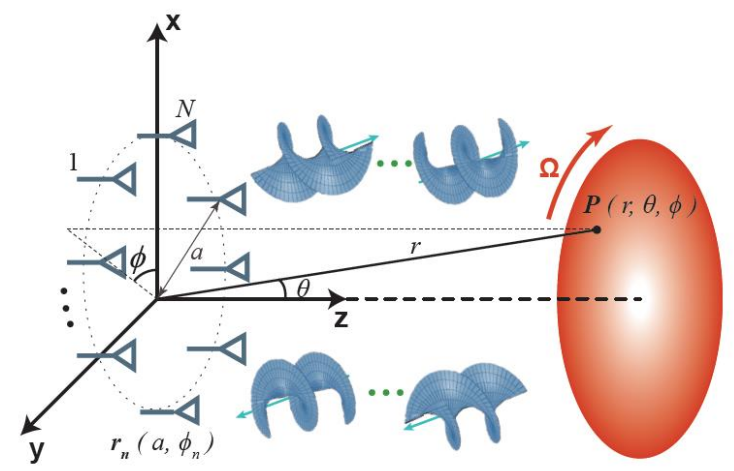

FIG. 1. Sketch map of the spinning object detection and imaging using OAM beams.

Since the amplitude modulation term $J_{l}(k a \sin \theta)$ in Eq. (1) will lead to negative effects on the object detection, it can be eliminated using the methods proposed in Ref. 28. Different from the design of array radii in the published paper, ${ }^{28}$ the frequency diversity technique ${ }^{31}$ is employed to design the beams with different OAM modes in this paper. This design can 
achieve a much simple and compact system, i.e., only one single-ring array is used. The designed signal $E_{s}\left(k_{l}, l\right)$ can be expressed in the formula

$$
\begin{aligned}
E_{s}\left(k_{l}, l\right) & =A_{l} \sum_{n=1}^{N} \frac{e^{-i k_{l}\left|\boldsymbol{r}-\boldsymbol{r}_{n}\right|} e^{i l \phi_{n}}}{\left|\boldsymbol{r}-\boldsymbol{r}_{n}\right|} \approx A_{l} \times \frac{e^{-i k_{l} r}}{r} \sum_{n=1}^{N} e^{i\left(\boldsymbol{k}_{l} \cdot \boldsymbol{r}_{n}+l \phi_{n}\right)} \\
& \approx \frac{e^{-i k_{l} r}}{r} i^{l} e^{i l \phi} N A_{l} J_{l}\left(k_{l} a \sin \theta\right)
\end{aligned}
$$

where $A_{l}$ and $k_{l}$ are the signal amplitude and wavenumber for the generation of the $l^{\text {th }}$ order OAM beam, which can be calculated by

$$
\begin{aligned}
A_{l} & =1 /[-0.0875 \ln (0.0198|l|-0.012)+0.1606] \\
k_{l} & =(1.0509|l|+1.1562) /\left(a \sin \theta_{0}\right)
\end{aligned}
$$

where $\theta_{0}$ is the designed radiation direction of the beam of the largest OAM mode number, which can be obtained by $\theta_{0}=\arcsin \left[\left(1.0509 l_{\max }+1.1562\right) /\left(k_{0} a\right)\right] . k_{0}$ is the wavenumber corresponding to the largest topological charge $\left(k_{0}=\lim _{l \rightarrow \max } k_{l}\right)$.

The radiation patterns for different OAM modes are depicted in Figure 2. In the simulation, the maximum mode number of the generated OAM beam is $l_{\max }=30$ and the corresponding frequency is set as $f_{0}=15 \mathrm{GHz}$. The array radius is $a=20 \lambda_{0}$, where $\lambda_{0}=2 \pi f_{0} / c$ is the wavelength and $c$ is the light speed in the vacuum. It is clear from Figure 2(a) that the radiation energy of different OAM modes cannot converge in the same direction and the maximum value varies with the mode number, which will lead to negative effects on the object detection and imaging. Based on Eq. (3), the amplitude and frequency of the excitation signal are designed, enabling the main lobes of different OAM modes converge in the same direction, shown in Figure 2(b).

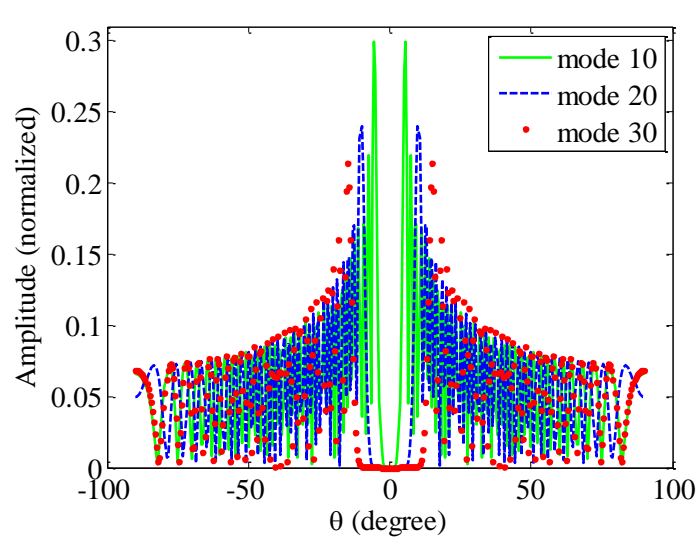

(a)

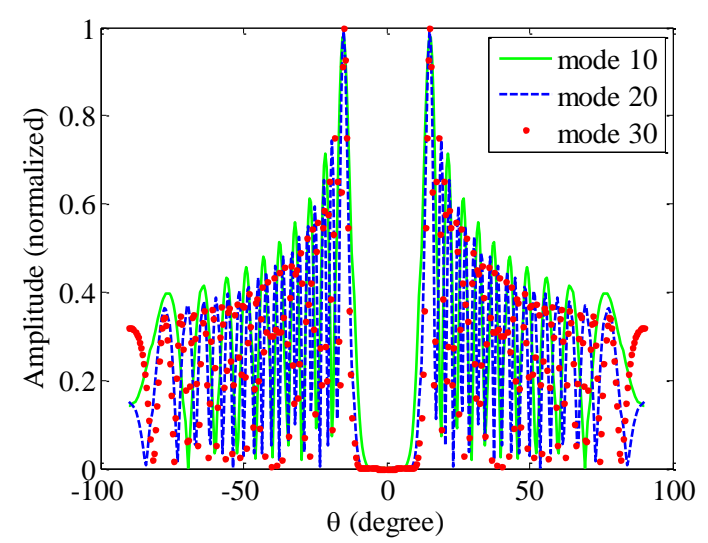

(b)

FIG. 2. Radiation patterns for different OAM modes $\left(\phi=0^{\circ}\right)$. (a) Operating at the same frequency, $f_{0}=15 \mathrm{GHz}$, (b) Designed patterns using the proposed method.

Due to the OAM-generation method and object's rotation feature, the imaging equation should be derived firstly. Generally, the UCA can be used to receive the echo scattered from the object. ${ }^{20}$ The phase term $\exp \left(i l \phi_{n}\right)$ and amplitude $A_{l}$ are multiplied by the echo signal received by the $n^{\text {th }}$ element, and then all the echoes are summed. Assuming that the object is composed of $M$ scattering points and the spinning velocity of the object is $\Omega$, the total echo $E_{r}\left(k_{l}, l\right)$ can be given by 


$$
\begin{aligned}
E_{r}\left(k_{l}, l\right) & =\sum_{m=1}^{M} \frac{N i^{l} e^{-i k_{l} r_{m}}}{r_{m}} \sigma_{m} e^{i l\left(\phi_{m}-\Omega t_{k}\right)} A_{l} J_{l}\left(k_{l} a \sin \theta_{m}\right) \sum_{n=1}^{N} \frac{e^{-i k_{l} r_{m}}}{r_{m}} A_{l} e^{i\left[k_{l} a \sin \theta_{m} \cos \left(\phi_{m}-\phi_{n}\right)+l \phi_{n}\right]} \\
& \approx \sum_{m=1}^{M} \frac{N^{2} i^{2 l} e^{-i 2 k_{l} r_{m}}}{r_{m}^{2}} \sigma_{m} e^{i l\left(2 \phi_{m}-\Omega t_{k}\right)} A_{l}^{2} J_{l}^{2}\left(k_{l} a \sin \theta_{m}\right) \\
& \approx N^{2} i^{2 l} e^{-i l \Omega t_{k}} \sum_{m=1}^{M} \frac{e^{-i 2 k_{l} r_{m}}}{r_{m}^{2}} \sigma_{m} e^{i 2 l \phi_{m}} A_{l}^{2} J_{l}^{2}\left(k_{l} a \sin \theta_{m}\right)
\end{aligned}
$$

where $\sigma_{m}$ denotes the radar cross section of the $m^{\text {th }}$ scattering point at the position $\left(r_{m}, \theta_{m}, \phi_{m}\right)$, $t_{k}=0, T_{r}, 2 T_{r}, \ldots, 2 l T_{r}$, and $T_{r}$ signifies the repetition period of the signal pulse.

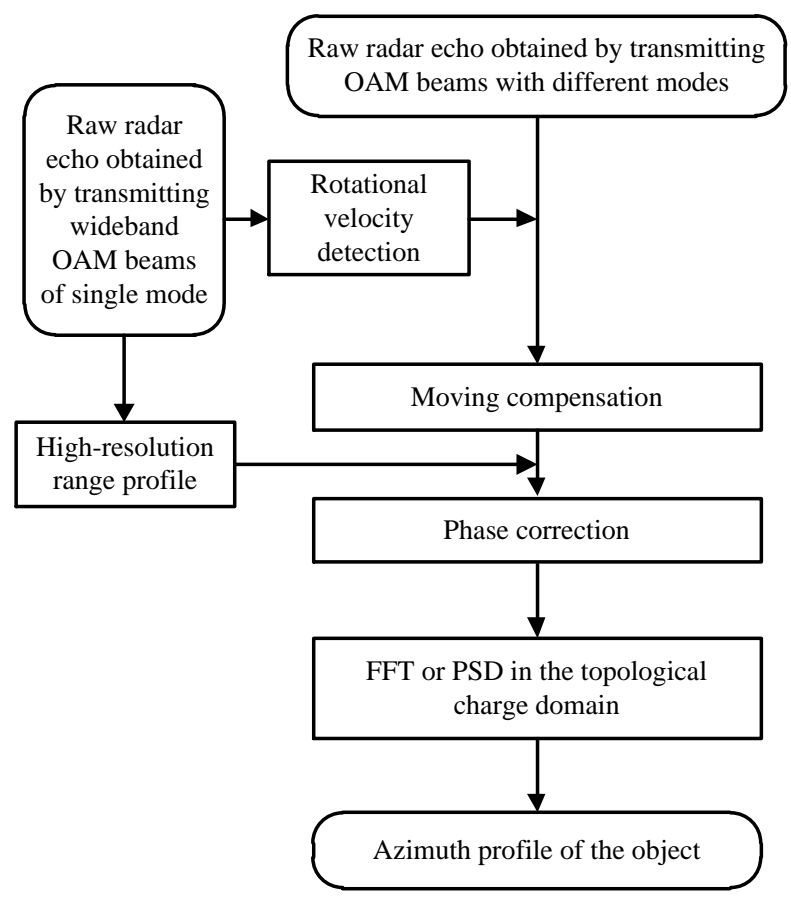

FIG. 3. Flowchart of the proposed imaging algorithm.

It can be seen from the imaging model in Eq. (4) that the azimuthal angle and the OAM mode number satisfy an approximate dual relation, which can be exploited to obtain the azimuthal information of the object. However, the rotational Doppler shift and the spinning velocity should be estimated firstly. Then, the moving compensation can be conducted and the phase correction caused by the frequency diversity are performed. Finally, FFT and other PSD estimation approaches can be applied to obtain the azimuthal profile of the object. The proposed imaging method of spinning object is illustrated in Figure 3.

To estimate the rotational velocity of the object, the signal phase difference $\Delta \varphi$ during the measurement time $t$ is observed. The Doppler frequency shift $\Delta f$ and the rotational velocity $\Omega$ can be calculated by the formula ${ }^{6,10}$

$$
\begin{aligned}
\Delta f & =\frac{(l+s) \Omega}{2 \pi} \\
\frac{\Delta f}{f} & =\frac{\Delta \varphi}{2 \pi l} \\
\Delta \varphi & =t l^{2} \Omega, t=n / f
\end{aligned}
$$

where $s$ is the mode number of spin angular momentum (SAM) and is equal to 0 for linearly 
polarized wave. $f$ is the frequency of the transmitted signal and $n$ indicates the number of sample periods. Eq. (5) indicates that the frequency shift can be multiplied by higher order OAM beams. Meanwhile, the phase difference $\Delta \varphi$ is $2 \pi l^{2}$, which is equivalent to 0 , if the time interval $t=T_{0}$, where $T_{0}$ is the rotation period of the object $\left(T_{0}=2 \pi / \Omega\right)$. Thus, a simple and fast method that can measure the time interval $t$ accurately is crucial for the detection of rotational velocity.

By transmitting wideband signal of a single OAM mode, the high-resolution range profile $r_{m}$ of the objects can be obtained. Therefore, the moving compensation and phase correction can be performed as follows

$$
E_{r}\left(k_{l}, l\right)=E_{r}\left(k_{l}, l\right) \times e^{i l \Omega t_{k}} \sum_{m=1}^{M} e^{i 2 k_{l} r_{m}}
$$

To validate the effectiveness of the proposed method, simulations are carried out, where the maximum topological charge applied in the imaging process is $l_{\max }=30$ and the corresponding frequency is set as $f_{0}=15 \mathrm{GHz}$. The OAM modes from -30 to 30 are used for the object imaging. The array radius is $a=0.4 \mathrm{~m}$ and the rotational velocity of the object is assumed to be $\Omega=50 \pi \mathrm{rad} / \mathrm{s}$. Two ideal scattering points are assumed to be at the positions $\left(500 \mathrm{~m}, 15^{\circ}, 0^{\circ}\right)$ and $\left(500 \mathrm{~m}, 15^{\circ}, 18^{\circ}\right)$, respectively. Comparison has been made between the simulation results before and after the moving compensation and phase correction was adopted. It can be seen from Figure 4(b) that two peaks occur at the expected positions $\phi=0 \mathrm{rad}$ and $\phi=0.1 \pi \mathrm{rad}$, whereas the objects cannot be identified correctly in Figure 4(a).

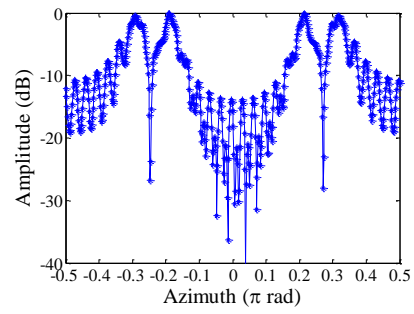

(a)

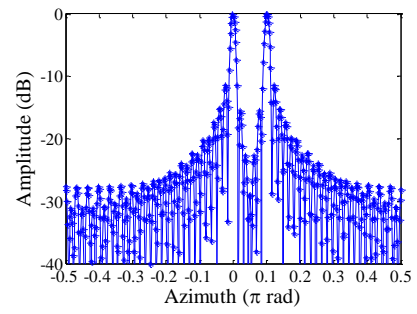

(b)

FIG. 4. Imaging results in the azimuthal dimension. (a) Without moving compensation or phase correction, (b) Results using the proposed method.

\section{EXPERIMENTAL RESULTS}

Based on the OAM-generating system developed in Refs. 21 and 27, a proof-of-concept experiment for the imaging of spinning object is carried out. The experimental setup is shown in Figure 5. A UCA is used to generate OAM beams with different modes, the circular metal plate acts as the object to be detected, and a horn antenna placed near the UCA is used to receive the echo signal. In the experiment, the main parameters are listed in Table 1. Since the distance between the antenna array and the object is not quite long, the object can be effectively illuminated by all the OAM beams with different modes. For simplicity, the carrier frequencies of the transmitted beams of different OAM modes are all set as $f=9.9 \mathrm{GHz}$. 


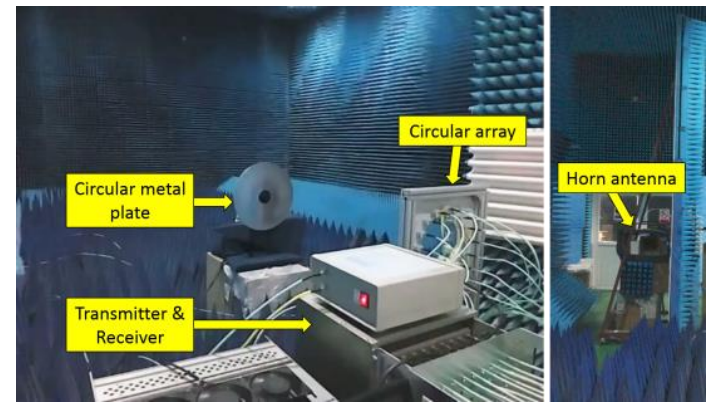

(a)

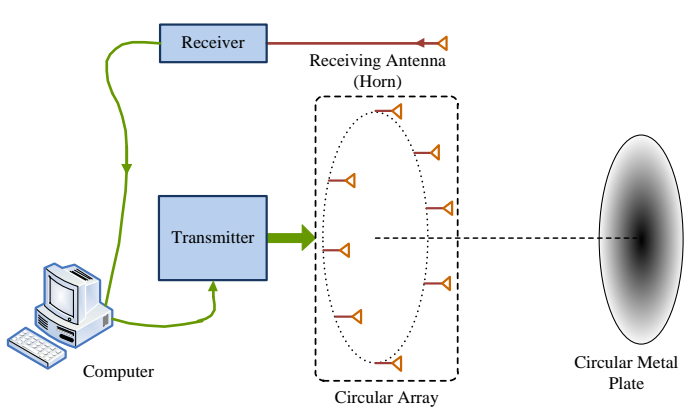

(b)

FIG. 5. Setup of the experimental scenario. (a) Photograph, (b) Schematic.

To estimate the rotational velocity of the object, a beam carrying OAM mode $l=1$ is transmitted to illuminate the object and the echo is sampled with the 1-ms interval. In the experiment, the object rotates at different velocities, i.e., $40 \pi \mathrm{rad} / \mathrm{s}$ and $50 \pi \mathrm{rad} / \mathrm{s}$, respectively. The sampled echo carrying rotational Doppler shift is displayed in Figure 6. Based on Eq. (5), the sample interval $t$ with phase difference $2 \pi$ should be estimated accurately, and thus the Doppler frequency shift and the rotational velocity can be obtained. In Figure 6(a), the average sample interval is $50 \mathrm{~ms}$, the frequency shift is $20 \mathrm{~Hz}$, and the rotational velocity is $40 \pi \mathrm{rad} / \mathrm{s}$. In Figure 6(b), the average sample interval is $40 \mathrm{~ms}$, the frequency shift is $25 \mathrm{~Hz}$, and the rotational velocity is $50 \pi \mathrm{rad} / \mathrm{s}$. The experimental imaging results in the azimuthal dimension are shown in Figure 7. Compared to the imaging result of static object in Figure $7(a),{ }^{21}$ the proposed imaging method can be used to reconstruct the spinning objects correctly.

TABLE I. Key parameters in the experiments.

\begin{tabular}{lll}
\hline \hline \multicolumn{1}{c}{ Symbol } & \multicolumn{1}{c}{ Quantity } & \multicolumn{1}{c}{ Value } \\
\hline$f$ & Frequency & $9.9 \mathrm{GHz}$ \\
$N$ & Number of antennas & 16 \\
$a$ & Radius of the circular array & $0.15 \mathrm{~m}$ \\
$a_{0}$ & Radius of the object & $0.25 \mathrm{~m}$ \\
$r$ & $\begin{array}{l}\text { Distance from the object to } \\
\text { the circular array }\end{array}$ & $2.1 \mathrm{~m}$ \\
& Rotational velocity of the & $40 \pi \mathrm{rad} / \mathrm{s}$, \\
$\Omega$ & object & $50 \pi \mathrm{rad} / \mathrm{s}$ \\
$l$ & Topological charge & {$[-7,7]$} \\
\hline \hline
\end{tabular}

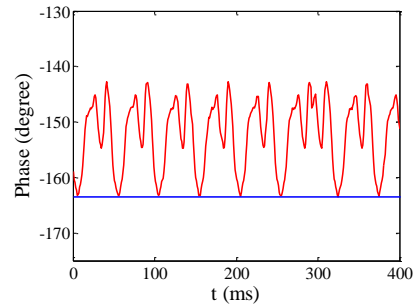

(a)

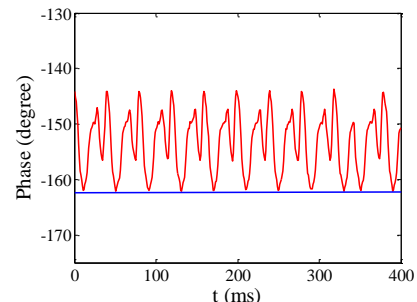

(b)

FIG. 6. Measured echo with OAM mode 1 (sampling time $400 \mathrm{~ms}$ ). (a) Rotational velocity, $40 \pi \mathrm{rad} / \mathrm{s}$, (b) Rotational velocity, $50 \pi \mathrm{rad} / \mathrm{s}$. 


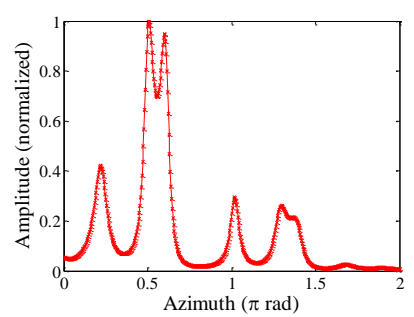

(a)

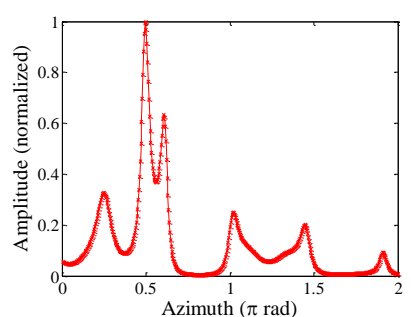

(b)

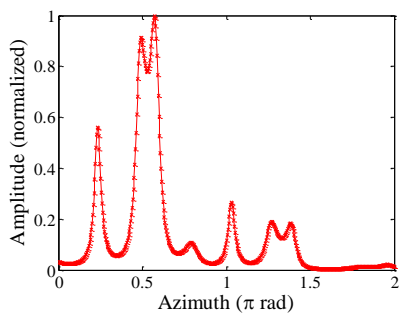

(c)

FIG. 7. Experimental results of the azimuthal profile. (a) Static object, (b) Rotational velocity, 40 $\pi \mathrm{rad} / \mathrm{s}$, (c) Rotational velocity, $50 \pi \mathrm{rad} / \mathrm{s}$.

\section{CONCLUSION}

In summary, the microwave imaging method of spinning object based on OAM has been studied. The frequency diversity principle was introduced to converge the OAM beams with different modes. The imaging model of spinning object was derived and the imaging approach was proposed, which was validated by both the simulations and proof-of-concept experiments. Results demonstrated that the proposed OAM-based imaging method of spinning objects can still be applied even when the LOS is perpendicular to the object. This work can facilitate the design and practice of OAM-based sensing systems.

\section{ACKNOWLEDGMENT}

This work was supported by the National Natural Science Foundation of China under Grant No. 61571011. The authors would like to thank Mr. S.N. Wang and the Shanghai Research Institute of Microwave Equipment for providing the experiment site and insightful discussions.

\section{REFERENCES}

${ }^{1}$ T. Asakura and N. Takai, "Dynamic laser speckles and their application to velocity measurements of the diffuse object,” Appl. Phys. A 25(3), 179-194 (1981).

${ }^{2}$ V.C. Chen, F. Li, and H. Wechsler, "Micro-Doppler effect in radar: phenomenon, model, and simulation study," IEEE Transactions on Aerospace and Electronic Systems 42(1), 2-21 (2006).

${ }^{3}$ X. Li, B. Deng, Y.L. Qin, H.Q. Wang, and Y.P. Li, "The influence of target micromotion on SAR and GMTI,” IEEE Transactions on Geoscience and Remote Sensing 49(7), 2738-2751 (2011).

${ }^{4}$ C. Clemente and J.J. Soraghan, "Vibrating target micro-Doppler signature in bistatic SAR with a fixed receiver," IEEE Transactions on Geoscience and Remote Sensing 50(8), 3219-3227 (2012).

${ }^{5}$ F. Tamburini, B. Thidé, G. Molina-Terriza, and G. Anzolin, "Twisting of light around rotating black holes," Nature Physics 7, 195-197 (2011). 
${ }^{6}$ M.P.J. Lavery, F.C. Speirits, S.M. Barnett, and M.J. Padgett, "Detection of a spinning object using light's orbital angular momentum," Science 341, 537-540 (2013).

${ }^{7}$ L. Allen, M. W. Beijersbergen, R. Spreeuw, and J. Woerdman, "Orbital angular momentum of light and the transformation of Laguerre-Gaussian laser modes,” Phys. Rev. A 45, 8185 (1992).

${ }^{8}$ A.M. Yao and M.J. Padgett, "Orbital angular momentum: origins, behavior and applications," Advances in Optics and Photonics 3(2), 161-204 (2011).

${ }^{9}$ N. Uribe-Patarroyo, A. Fraine, D.S. Simon, L. Minaeva, and A.V. Sergienko, "Object identification using correlated orbital angular momentum states," Physical Review A 110, 043601 (2013).

${ }^{10}$ M.Y. Zhao, X.L. Gao, M.T. Xie, W.S. Zhai, W.J. Xu, S.G. Huang, and W.Y. Gu, "Measurement of the rotational Doppler frequency shift of a spinning object using a radio frequency orbital angular momentum beam," Optics Letters 41(11), 2549-2552 (2016).

${ }^{11}$ A.E. Willner, H. Huang, Y. Yan, Y. Ren, N. Ahmed, G. Xie, C. Bao, L. Li, Y. Cao, Z. Zhao, J. Wang, M.P.J. Lavery, M. Tur, S. Ramachandran, A.F. Molisch, N. Ashrafi, and S. Ashrafi, "Optical communications using orbital angular momentum beams," Advances in Optics and Photonics 7(1), 66-106 (2015).

${ }^{12}$ J. Wang, "Advances in communications using optical vortices," Photonics Research 4(5), B14-B28 (2016).

${ }^{13}$ J. Wang, "Data information transfer using complex optical fields: a review and perspective," Chinese Optics Letters 15(3), 030005 (2017).

${ }^{14}$ A.E. Willner, J. Wang, and H. Huang, “A different angle on light communications," Science 337(6095), 655-656 (2012).

${ }^{15}$ A. Wang, L. Zhu, S. Chen, C. Du, Q. Mo, and J. Wang, "Characterization of LDPC-coded orbital angular momentum modes transmission and multiplexing over a 50-km fiber," Optics Express 24(11), 11716-11726 (2016).

${ }^{16}$ F. Tamburini, E. Mari, A. Sponselli, B. Thidé, A. Bianchini, and F. Romanato, "Encoding many channels on the same frequency through radio vorticity: first experimental test," New Journal of Physics 14, 033001 (2012).

${ }^{17}$ N.B. Zhao, X.Y. Li, G.F. Li, and J.M. Kahn, "Capacity limits of spatially multiplexed free-space communication," Nature Photonics 9, 822-826 (2015).

${ }^{18}$ Z.F. Zhang, S.L. Zheng, Y.L. Chen, X.F. Jin, H. Chi, and X.M. Zhang, "The capacity gain of orbital angular momentum based multiple-input-multiple-output system,” Scientific Reports 6, 25418 (2016).

${ }^{19} \mathrm{~L}$. Li and F. Li, "Beating the Rayleigh limit: Orbital-angular-momentum-based super-resolution diffraction tomography,” Physical Review E 88, 033205 (2013).

${ }^{20}$ K. Liu, Y.Q. Cheng, Z.C. Yang, H.Q. Wang, Y.L. Qin, and X. Li, "Orbital-angular-momentum-based electromagnetic vortex imaging," IEEE Antennas and Wireless Propagation Letters 14, 711-714 (2015).

${ }^{21}$ K. Liu, Y.Q. Cheng, Y. Gao, X. Li, Y.L. Qin, and H.Q. Wang, "Super-resolution radar imaging based on experimental OAM beams," Applied Physics Letters 110, 164102 (2017).

${ }^{22}$ Y.L. Chen, S.L. Zheng, X.F. Jin, H. Chi, and X.M. Zhang, "Single-frequency computational imaging using OAM-carrying electromagnetic wave," Journal of Applied Physics 121, 184506 (2017). 
${ }^{23}$ M.L. Chen, L.J. Jiang, and W. Sha, "Artificial perfect electric conductor-perfect magnetic conductor anisotropic metasurface for generating orbital angular momentum of microwave with nearly perfect conversion efficiency,” Journal of Applied Physics 119, 064506 (2016).

${ }^{24}$ S.X. Yu, L. Li, G.M. Shi, C. Zhu, and Y. Shi, "Generating multiple orbital angular momentum vortex beams using a metasurface in radio frequency domain,” Applied Physics Letters 108, 241901 (2016).

${ }^{25}$ J.J. Jin, J. Luo, X.H. Zhang, H. Gao, X. Li, M.B. Pu, P. Gao, Z.Y. Zhao, and X.G. Luo, "Generation and detection of orbital angular momentum via metasurface," Scientific Reports 6, 24286 (2016).

${ }^{26}$ S.L. Zheng, X.N. Hui, X.F. Jin, H. Chi, and X.M. Zhang, "Transmission characteristics of a twisted radio wave based on circular traveling-wave antenna," IEEE Transactions on Antennas and Propagation 63(4), 1530-1536 (2015).

${ }^{27}$ K. Liu, H.Y. Liu, Y.L. Qin, Y.Q. Cheng, S.N. Wang, X. Li, and H.Q. Wang, "Generation of OAM beams using phased array in the microwave band," IEEE Transactions on Antennas and Propagation 64(9), 3850-3857 (2016).

${ }^{28}$ K. Liu, Y.Q. Cheng, X. Li, Y.L. Qin, H.Q. Wang, and Y.W. Jiang, "Generation of orbital angular momentum beams for electromagnetic vortex imaging," IEEE Antennas and Wireless Propagation Letters 15, 1873-1876 (2016).

${ }^{29}$ B. Thidé, H. Then, J. Sjöholm, K. Palmer, J. Bergman, T.D. Carozzi, Y.N. Istomin, N.H. Ibragimov, and R. Khamitova, "Utilization of photon orbital angular momentum in the low-frequency radio domain," Physical Review Letters 99, 087701 (2007).

${ }^{30}$ S.M. Mohammadi, L.K.S. Daldorff, J.E.S. Bergman, R.L. Karlssson, B. Thidé, K. Forozesh, T.D. Carozzi, and B. Isham, "Orbital angular momentum in radio- A system study," IEEE Transactions on Antennas and Propagation 58(2), 565-572 (2010).

${ }^{31}$ P.F. Sammartino, C.J. Baker, and H. Griffiths, "Frequency diverse MIMO techniques for radar," IEEE Transactions on Aerospace and Electronic Systems 49(1), 201-222 (2013). 